\title{
Determination of Single Cell Growth Kinetics with Microcantilever Sensors
}

\author{
Aleksandra Wańczyk, Bogdan Łabędź, and Zenon Rajfur \\ Faculty of Physics, Astronomy and Applied Computer Science, Jagiellonian University \\ prof. Stanisława Łojasiewicza 11, Krakow, Poland \\ aleksandra.dejko@uj.edu.pl; bogdan2.labedz@doctoral.uj.edu.pl; zenon.rajfur@uj.edu.pl
}

\section{Extended Abstract}

Study of different physical and biochemical parameters of a cell during its life cycle and analysis of underlying regulatory processes are crucial in biomedical and life sciences [1,2]. One of such parameters is a mass of the cell which is inherently associated with important cellular processes like growth or reproduction. Despite decades of research on cell cycle, there is still a great need for better understanding of many involved process on a single cell level. Such measurements are aided by nano and micro electromechanical systems (NEMS/MEMS) to which nano- and microcantilever biosensors belong to. Cantilever-based devices convert biological or chemical interactions into mechanical response such as cantilever bending amplitude or its resonance frequency change.

Objective of this study was the establishment of experimental procedures and analytical tools for comprehensive studies of single cell life cycle using microcantilever biosensors. This work investigated growth kinetics of single cells of Saccharomyces cerevisiae (Instaferm, Lallemand, Poland). S. cerevisiae are commonly used in the cell studies as a model eukaryotic cell. They are widely available and easy to maintain. Here, we demonstrate that it is possible to measure mass changes of single cell during the cell budding using micro- cantilever biosensor. Analysis of mass changes was based on measuring cantilever resonance frequency changes due to cells adhesion and reproduction. Another important factor in this analysis was the determination of individual cell positions along the cantilever length [3]. Their positions were identified

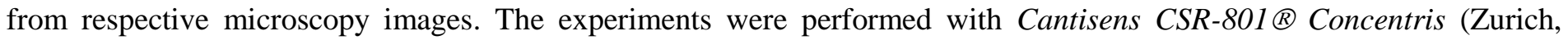
Switzerland) cantilever biosensor and Axio Observer Z1 Zeiss (Jena, Germany) microscope.

The results of our study provide basic groundwork for further studies of single cell cycle parameters. This work also shows that combination of microcantilever-based sensors and microscopy techniques can be a powerful tool in cell mass kinetics analysis at a single cell level.

\section{References}

[1] M. Bache, R. Taboryski, S. Schmid, J. Aamand, and M. H. Jakobsen, "Investigations on antibody binding to a microcantilever coated with a BAM pesticide residue," PNAS, vol. 6, pp. 386, 2011.

[2] K. Park, L. J. Millet, N. Kim, H. Li, X. Jin, G. Popescu, N. R. Aluru, K. J. Hsia, and R. Bashir, "Measurement of adherent cel mass and growth," PNAS, pp. 20691 - 20696, 2010.

[3] S. Dohn, W. Svendsen, and A. Boisen, "Mass and position determination of attached particles on cantilever based mass sensors," Rev. Sci. Instrum., vol. 78, 2007. 